\title{
Path Planning in a Riemannian Manifold using Optimal Control
}

\author{
Souma Mazumdar* \\ Department of Theoretical Sciences \\ S. N. Bose National Centre for Basic Sciences \\ Block - JD, Sector - III, Salt Lake City, Kolkata - 700106
}

\begin{abstract}
We consider the motion planning of an object in a Riemannian manifold where the object is steered from an initial point to a final point utilizing optimal control. Considering Pontryagin Minimization Principle we compute the Optimal Controls needed for steering the object from initial to final point. The Optimal Controls were solved with respect to time $t$ and shown to have norm 1 which should be the case when the extremal trajectories, which are the solutions of Pontryagin Principle, are arc length parametrized. The extremal trajectories are supposed to be the geodesics on the Riemannian manifold. So we compute the geodesic curvature and the Gaussian curvature of the Riemannian structure.
\end{abstract}

Keywords: Motion Planning, Optimal Control, Geometric Control Theory, Riemannian Manifold, Riemannian Curvature, Nonlinear Differential Equation

*Email: souma.mazumdar@bose.res.in, Phone: 09903144810 


\section{Introduction}

Path Planning or Motion Planning is today an active area of research in the control theory community which concerns about steering an object from one point to another utilizing controls. Practical application of this is abound in real life scenario. Starting from Optimal path planning of a mobile robot in an unknown terrain to proper manouevering of an autonomous vehicle to landing of an aircraft in an airport. Utilizing the Optimal Control Theory and Pontryagin Principle is one of the tools used by Control theorists to deal with motion planning problems. While on the other hand there are other techniques employed in motion planning problems like the continuation method as shown by Sussman. All of these methods concerns about finding a proper control which can steer the object from one point to another. Sussman in his work [1] discussed about a number of techniques of motion planning all of which are different in the mathematical ideas they involve. One of them is the Continuation method where the mathematical idea goes like lifting the problem of motion planning from the state space to the control space and then solve a differential equation to find the controls. A number of interesting papers [2, 3, 4] have been written along this line by Sussman and Chitour. We will not go far discussing about various methods of motion planning but come back to the topic of this paper which is motion planning in a Riemannian manifold utilizing optimal controls.

Most of the problems in control theory fall under the purview of SubRiemannian Geometry. While speaking about Sub-Riemannian Geometry we think of a underlying distribution $\mathcal{D}$ which is a subspace of the the tangent space $T_{q} M$ at a point $q$, where $q \in M, M$ being the manifold. Here the vector 
fields span a subspace of the tangent space but if the vector fields form a bracket generating family then the vector fields together with their Lie brackets span the whole tangent space. But in our problem we speak of Riemannian manifold. The prime difference in the Riemannian case from the Sub-Riemannian problem is that the vector fields span the whole tangent space. We deal with the problem of motion planning in a Riemannian manifold using the principles of Optimal Control. Moreover the manifold being Riemannian, it has an intrinsic curvature. So Optimal Control problems in a Riemannian manifold will be very different from other cases. In Sub-Riemannian case the extremal curves which are the solutions of Pontryagin Principle can be both Normal and Abnormal extremals. But in Riemannian case as shown by Agrachev 5$]$ the extremals are always Normal extremals. So in our work we are primarily concerned with Normal extremals as Abnormal extremals do not exist in a Riemannian manifiold.

In our problem we consider the manifold to be a $2-D$ Riemannian manifold. So the control problem has two orthogonal vector fields which span the tangent space $T_{q} M$ at a point $q \in M$. The family of vector fields can be involutive, that is their Lie bracket generates 0 or may be non involutive. We apply the principles of Optimal Control theory and Pontryagin Principle to find the Optimal Controls which help in steering the object from one point to another on the manifold. Further we assume, the normal extremals which are the solutions of Pontryagin principle are arc length parametrized. So the velocity at any point on the trajectory is always 1 . As the manifold possesses an intrinsic curvature, in addition to computing the Optimal Controls, we find the geodesic and Gaussian curvatures. The Gaussian curvature evaluates to 0 which should be the case in case of involutive vector fields [5] as the Lie bracket of our chosen family of orthogonal vector fields generates 0 . 


\section{Problem Formulation}

We consider a $2-D$ Riemannian manifold. The vectors fields span the whole tangent space. So in our problem we consider two vector fields which form an orthogonal frame. A control system on such a manifold is described by the following equation.

$$
\dot{q}=u_{1}\left[\begin{array}{l}
q_{1} \\
q_{2}
\end{array}\right]+u_{2}\left[\begin{array}{c}
q_{2} \\
-q_{1}
\end{array}\right]
$$

where $u_{1}$ and $u_{2}$ are the controls. Note that the vector fields $\left[\begin{array}{c}q_{1} \\ q_{2}\end{array}\right]$ and $\left[\begin{array}{c}q_{2} \\ -q_{1}\end{array}\right]$ form an orthogonal frame. We cast the problem in the Pontryagin Principle formalism and solve for the Optimal Controls. Pontryagin Principle is a Hamiltonian formalism. So it generates differential equations of position $(q)$ and momentum $(p)$. In our case also we are confronted with such differential equations which are nonlinear and hard to solve. But with the help of the fact that the velocity at any point of the extremal curves is always 1 we were able to solve these equations with ingenuity.

\section{Pontryagin Principle and Hamiltonian Formal- ism}

We consider spacial optimality and time to vary between 0 and 1 that is $t \in$ $(0,1)$. The objective function to be optimized is the length functional $\int_{0}^{1} \sqrt{u_{1}^{2}+u_{2}^{2}}$ which is same as optimizing the energy functional $\frac{1}{2} \int_{0}^{1}\left(u_{1}^{2}+u_{2}^{2}\right)$. We cast the problem is the Pontryagin Formalism. The associated Hamiltonian is con- 
structed as follows

$$
H(p, q, u)=-\frac{1}{2}\left(u_{1}^{2}+u_{2}^{2}\right)+p^{T}\left(u_{1} f_{1}(q)+f_{2}(q)\right)
$$

where $f_{1}(q)=\left[\begin{array}{l}q_{1} \\ q_{2}\end{array}\right]$ and $f_{2}(q)=\left[\begin{array}{c}q_{2} \\ -q_{1}\end{array}\right]$ and $p^{T}$ stands for the transpose of the momentum covector. Here $q=\left[\begin{array}{c}q_{1} \\ q_{2}\end{array}\right]$ and $p=\left[\begin{array}{c}p_{1} \\ p_{2}\end{array}\right]$.

Minimizing the above Hamiltonian with respect to the controls we obtain the controls as

$$
\begin{aligned}
& u_{1}=<p, f_{1}(q)> \\
& u_{2}=<p, f_{2}(q)>
\end{aligned}
$$

where $<., .>$ defines the inner product between the vectors and the covectors. The Hamilton's equations are given by the following

$$
\begin{gathered}
\dot{q}=<p, f_{1}(q)>f_{1}(q)+<p, f_{2}(q)>f_{2}(q) \\
\dot{p}=-<p, f_{1}(q)><p, D_{q} f_{1}(q)>-<p, f_{2}(q)><p, D_{q} f_{2}(q)>
\end{gathered}
$$

where $D_{q}$ stands for the Jacobian.

The momentum covector written as above is a row vector. When written in column vector form it takes the form,

$$
\dot{p}=-<p, f_{1}(q)><D_{q} f_{1}(q), p>-<p, f_{2}(q)><D_{q} f_{2}(q), p>
$$


When written out in components representation (4) takes the form,

$$
\begin{aligned}
& \dot{q}_{1}=\left(p_{1} q_{1}+p_{2} q_{2}\right) q_{1}+\left(p_{1} q_{2}-p_{2} q_{1}\right) q_{2} \\
& \dot{q}_{2}=\left(p_{1} q_{1}+p_{2} q_{2}\right) q_{2}-\left(p_{1} q_{2}-p_{2} q_{1}\right) q_{1}
\end{aligned}
$$

For finding the equation for momentum covectors we compute the respective Jacobians. Now,

$$
D_{q} f_{1}(q) p=\left[\begin{array}{ll}
1 & 0 \\
0 & 1
\end{array}\right]\left[\begin{array}{l}
p_{1} \\
p_{2}
\end{array}\right]=\left[\begin{array}{l}
p_{1} \\
p_{2}
\end{array}\right]
$$

and

$$
D_{q} f_{2}(q) p=\left[\begin{array}{cc}
0 & 1 \\
-1 & 0
\end{array}\right]\left[\begin{array}{l}
p_{1} \\
p_{2}
\end{array}\right]=\left[\begin{array}{c}
p_{2} \\
-p_{1}
\end{array}\right]
$$

Therefore (5) when written out in components representation takes the form,

$$
\begin{aligned}
& \dot{p}_{1}=-\left(p_{1} q_{1}+p_{2} q_{2}\right) p_{1}-\left(p_{1} q_{2}-p_{2} q_{1}\right) p_{2} \\
& \dot{p}_{2}=-\left(p_{1} q_{1}+p_{2} q_{2}\right) p_{2}+\left(p_{1} q_{2}-p_{2} q_{1}\right) p_{1}
\end{aligned}
$$

When simplifield the 4 differential equations of Hamilton's equation can be written as follows

$$
\begin{aligned}
& \dot{q}_{1}=p_{1}\left(q_{1}^{2}+q_{2}^{2}\right) \\
& \dot{q}_{2}=p_{2}\left(q_{1}^{2}+q_{2}^{2}\right) \\
& \dot{p}_{1}=-q_{1}\left(p_{1}^{2}-p_{2}^{2}\right)-2 p_{1} p_{2} q_{2} \\
& \dot{p}_{2}=-q_{2}\left(p_{2}^{2}-p_{1}^{2}\right)-2 p_{1} p_{2} q_{1}
\end{aligned}
$$

Moreover we consider the normal extremals are arc length parametrized. Therefore the velocity at any point of the trajectory is always 1 . So we have the 
additional equation,

$$
\dot{q}_{1}^{2}+\dot{q}_{2}^{2}=1
$$

\section{Solve of the Hamilton's Equations}

From the $1 s t$ and $2 n d$ equations of the set(11) we have

$$
\begin{aligned}
& p_{1}=\frac{\dot{q}_{1}}{q_{1}^{2}+q_{2}^{2}} \\
& p_{2}=\frac{\dot{q}_{2}}{q_{1}^{2}+q_{2}^{2}}
\end{aligned}
$$

Differentaiting the above equations with respect to time we have,

$$
\begin{aligned}
& \dot{p}_{1}=\frac{\left(q_{1}^{2}+q_{2}^{2}\right) \ddot{q}_{1}-\dot{q}_{1}\left(2 q_{1} \dot{q}_{1}+2 q_{2} \dot{q}_{2}\right)}{\left(q_{1}^{2}+q_{2}^{2}\right)^{2}} \\
& \dot{p}_{2}=\frac{\left(q_{1}^{2}+q_{2}^{2}\right) \ddot{q}_{2}-\dot{q}_{2}\left(2 q_{1} \dot{q}_{1}+2 q_{2} \dot{q}_{2}\right)}{\left(q_{1}^{2}+q_{2}^{2}\right)^{2}}
\end{aligned}
$$

Puting the values of $\dot{p}_{1}$ and $\dot{p}_{2}$ in the $3 r d$ and 4 th equations of the set (11) and simplifying we have,

$$
\begin{aligned}
& \left(q_{1}^{2}+q_{2}^{2}\right) \ddot{q}_{1}-\dot{q}_{1}\left(2 q_{1} \dot{q}_{1}+2 q_{2} \dot{q}_{2}\right)=-q_{1}\left(\dot{q}_{1}^{2}-\dot{q}_{2}^{2}\right)-2 \dot{q}_{1} \dot{q}_{2} q_{2} \\
& \left(q_{1}^{2}+q_{2}^{2}\right) \ddot{q}_{2}-\dot{q}_{2}\left(2 \dot{q}_{1} q_{1}+2 \dot{q}_{2} q_{2}\right)=-q_{2}\left(\dot{q}_{2}^{2}-\dot{q}_{1}^{2}\right)-2 \dot{q}_{1} \dot{q}_{2} q_{1}
\end{aligned}
$$

Multiplying equation (15) by $\dot{q}_{1}$ and equation (16) by $\dot{q}_{2}$ and adding them we have,

$$
\begin{aligned}
& \left(q_{1}^{2}+q_{2}^{2}\right)\left(\dot{q}_{1} \ddot{q}_{1}+\dot{q}_{2} \ddot{q}_{2}\right)-\left(2 q_{1} \dot{q}_{1}+2 q_{2} \dot{q}_{2}\right)\left(\dot{q}_{1}^{2}+\dot{q}_{2}^{2}\right) \\
& =-\dot{q}_{1} q_{1}\left(\dot{q}_{1}^{2}-\dot{q}_{2}^{2}\right)-\dot{q}_{2} q_{2}\left(\dot{q}_{2}^{2}-\dot{q}_{1}^{2}\right)-2 \dot{q}_{1}^{2} \dot{q}_{2} q_{2}-2 \dot{q}_{2}^{2} \dot{q}_{1} q_{1}
\end{aligned}
$$


Now we have the additional equation,

$$
\dot{q}_{1}^{2}+\dot{q}^{2}=1
$$

Differentiating the above equation with respect to time and dividing by 2 we have

$$
\dot{q}_{1} \ddot{q}_{1}+\dot{q}_{2} \ddot{q}_{2}=0
$$

Now using equations (18) and (19) and puting in equation (17) we have,

$$
-\left(2 q_{1} \dot{q}_{1}+2 q_{2} \dot{q}_{2}\right)=-\dot{q}_{1} q_{1}\left(\dot{q}_{1}^{2}-\dot{q}_{2}^{2}\right)-\dot{q}_{2} q_{2}\left(\dot{q}_{2}^{2}-\dot{q}_{1}^{2}\right)-2 \dot{q}_{1}^{2} \dot{q}_{2} q_{2}-2 \dot{q}_{2}^{2} \dot{q}_{1} q_{1}
$$

Simplifying the above equation we have,

$$
-2 q_{1} \dot{q}_{1}-2 q_{2} \dot{q}_{2}=-\left(\dot{q}_{1} q_{1}+\dot{q}_{2} q_{2}\right)\left(\dot{q}_{1}^{2}+\dot{q}_{2}^{2}\right)
$$

Again using (18) we have the above equation as

$$
-2 q_{1} \dot{q}_{1}-2 q_{2} \dot{q}_{2}=-\dot{q}_{1} q_{1}-\dot{q}_{2} q_{2}
$$

which simplifies to

$$
\dot{q}_{1} q_{1}+\dot{q}_{2} q_{2}=0
$$

Integrating the above equation with respect to time we have,

$$
q_{1}^{2}+q_{2}^{2}=K
$$


where $K$ is the constant of integration. The above constant can be scaled to 1 . Then we have the above equation as,

$$
q_{1}^{2}+q_{2}^{2}=1
$$

Using this relation for the $1 s t$ and $2 n d$ equations of the set (11) we have,

$$
\begin{aligned}
& \dot{q}_{1}=p_{1} \\
& \dot{q}_{2}=p_{2}
\end{aligned}
$$

Differentiating the above set with respect to time we have,

$$
\begin{aligned}
& \dot{p}_{1}=\ddot{q}_{1} \\
& \dot{p}_{2}=\ddot{q}_{2}
\end{aligned}
$$

Now using equations (26) and (27) and puting in the $3 r d$ and $4 t h$ equations of the set (11) we have,

$$
\begin{aligned}
& \ddot{q}_{1}=-q_{1}\left(\dot{q}_{1}^{2}-\dot{q}_{2}^{2}\right)-2 \dot{q}_{1} \dot{q}_{2} q_{2} \\
& \ddot{q}_{2}=-q_{2}\left(\dot{q}_{2}^{2}-\dot{q}_{1}^{2}\right)-2 \dot{q}_{1} \dot{q}_{2} q_{1}
\end{aligned}
$$

Now multiplying equation (28) by $q_{2}$ and equation (29) by $q_{1}$ and adding them and then simplifying we have,

$$
q_{2} \ddot{q}_{1}+q_{1} \ddot{q}_{2}=-2 \dot{q}_{1} \dot{q}_{2}\left(q_{1}^{2}+q_{2}^{2}\right)
$$

Now using equation (25) we have the above equation as

$$
q_{2} \ddot{q}_{1}+q_{1} \ddot{q}_{2}=-2 \dot{q}_{1} \dot{q}_{2}
$$


Dividing both sides by $\dot{q}_{1} \dot{q}_{2}$ we have the above equation as,

$$
\frac{q_{2} \ddot{q}_{1}}{\dot{q}_{1} \dot{q}_{2}}+\frac{q_{1} \ddot{q}_{2}}{\dot{q}_{1} \dot{q}_{2}}=-2
$$

Now from equation (19) we have

$$
\frac{\ddot{q}_{1}}{\dot{q}_{2}}=-\frac{\ddot{q}_{2}}{\dot{q}_{1}}
$$

Using the above equation we have equation (32) as

$$
\frac{q_{2}}{\dot{q}_{1}}\left(-\frac{\ddot{q}_{2}}{\dot{q}_{1}}\right)+\frac{q_{1} \ddot{q}_{2}}{\dot{q}_{1} \dot{q}_{2}}=-2
$$

which on simplifying gives,

$$
\frac{\ddot{q}_{2}}{\dot{q}_{1}}\left(\frac{q_{1}}{\dot{q}_{2}}-\frac{q_{2}}{\dot{q}_{1}}\right)=-2
$$

Now from equation (23) we have,

$$
\frac{q_{1}}{\dot{q}_{2}}=-\frac{q_{2}}{\dot{q}_{1}}
$$

Using the above equation and puting in equation (35) we have

$$
2 \frac{\ddot{q}_{2}}{\dot{q}_{1}} \frac{q_{1}}{\dot{q}_{2}}=-2
$$

which on simplifying gives

$$
\frac{\ddot{q}_{2}}{\dot{q}_{2}}=-\frac{\dot{q}_{1}}{q_{1}}
$$

Now the above equation can be written as,

$$
\frac{d}{d t}\left(\ln \dot{q}_{2}\right)=-\frac{d}{d t}\left(\ln q_{1}\right)
$$


which on integrating gives,

$$
\begin{aligned}
& \ln \dot{q}_{2}=-\ln q_{1}+\ln C_{1} \\
& \Longrightarrow \dot{q}_{2}=\frac{C_{1}}{q_{1}}
\end{aligned}
$$

where $C_{1}$ is the constant of integration.

Puting the above value of $\dot{q}_{2}$ in the equation (18) we have,

$$
\dot{q}_{1}^{2}+\frac{C_{1}^{2}}{q_{1}^{2}}=1
$$

Therefore,

$$
\begin{aligned}
& \dot{q}_{1}=\sqrt{1-\frac{C_{1}^{2}}{q_{1}^{2}}} \\
& \Longrightarrow \frac{d q_{1}}{d t}=\sqrt{1-\frac{C_{1}^{2}}{q_{1}^{2}}}
\end{aligned}
$$

Therefore,

$$
\begin{aligned}
& \frac{d q_{1}}{\sqrt{1-\frac{C_{1}^{2}}{q_{1}^{2}}}}=d t \\
& \Longrightarrow \frac{q_{1} d q_{1}}{\sqrt{q_{1}^{2}-C_{1}^{2}}}=d t
\end{aligned}
$$

Integrating the above equation we have,

$$
\begin{aligned}
& \sqrt{q_{1}^{2}-C_{1}^{2}}=t+C_{2} \\
& \Longrightarrow q_{1}=\sqrt{\left(t+C_{2}\right)^{2}+C_{1}^{2}}
\end{aligned}
$$


where $C_{2}$ is the constant of integration.

From equation (40) we have,

$$
\begin{aligned}
& \dot{q}_{2}=\frac{C_{1}}{\sqrt{\left(t+C_{2}\right)^{2}+C_{1}^{2}}} \\
& \Longrightarrow d q_{2}=\frac{C_{1} d t}{\sqrt{\left(t+C_{2}\right)^{2}+C_{1}^{2}}}
\end{aligned}
$$

Now for integration of the right side of the above equation we substitute $t+C_{2}=$ $C_{1} \tan \theta$. Therefore by this substitution (45) takes the form,

$$
d q_{2}=C_{1} \sec \theta d \theta
$$

Integrating both sides we have,

$$
q_{2}=C_{1}[\ln (\sec \theta+\tan \theta)]+C_{3}
$$

where $C_{3}$ is the constant of integration.

Substituting back the value of $\sec \theta$ and $\tan \theta$ with respect to $t$ we have,

$$
q_{2}=C_{1}\left[\ln \left(\frac{t+C_{2}+\sqrt{\left(t+C_{2}\right)^{2}+C_{1}^{2}}}{C_{1}}\right)\right]+C_{3}
$$

Now from equations (26) we have,

$$
p_{1}=\dot{q}_{1}=\frac{t+C_{2}}{\sqrt{\left(t+C_{2}\right)^{2}+C_{1}^{2}}}
$$

and,

$$
p_{2}=\dot{q}_{2}=\frac{C_{1}}{\sqrt{\left(t+C_{2}\right)^{2}+C_{1}^{2}}}
$$


Therefore we have the position momentum $(q, p)$ set as,

$$
\begin{aligned}
& q_{1}=\sqrt{\left(t+C_{2}\right)^{2}+C_{1}^{2}} \\
& q_{2}=C_{1}\left[\ln \left(\frac{t+C_{2}+\sqrt{\left(t+C_{2}\right)^{2}+C_{1}^{2}}}{C_{1}}\right)\right]+C_{3} \\
& p_{1}=\frac{t+C_{2}}{\sqrt{\left(t+C_{2}\right)^{2}+C_{1}^{2}}} \\
& p_{2}=\frac{C_{1}}{\sqrt{\left(t+C_{2}\right)^{2}+C_{1}^{2}}}
\end{aligned}
$$

\section{Computaion of Optimal Controls}

We have from equations (3),

$$
\begin{aligned}
& u_{1}=<p, f_{1}(q)> \\
& u_{2}=<p, f_{2}(q)>
\end{aligned}
$$

The 1 st equation of the above set implies

$$
\begin{aligned}
u_{1}=p^{T} f_{1}(q) & =\left[\begin{array}{ll}
p_{1} & p_{2}
\end{array}\right]\left[\begin{array}{l}
q_{1} \\
q_{2}
\end{array}\right]=p_{1} q_{1}+p_{2} q_{2} \\
& =t+C_{2}+\frac{C_{1}}{\sqrt{\left(t+C_{2}\right)^{2}+C_{1}^{2}}}\left[C_{1}\left[\ln \left(\frac{t+C_{2}+\sqrt{\left(t+C_{2}\right)^{2}+C_{1}^{2}}}{C_{1}}\right)\right]+C_{3}\right]
\end{aligned}
$$

The $2 n d$ equation of the above set implies

$$
\begin{aligned}
u_{2}=p^{T} f_{2}(q) & =\left[\begin{array}{ll}
p_{1} & p_{2}
\end{array}\right]\left[\begin{array}{c}
q_{2} \\
-q_{1}
\end{array}\right]=p_{1} q_{2}-p_{2} q_{1} \\
& =\frac{t+C_{2}}{\sqrt{\left(t+C_{2}\right)^{2}+C_{1}^{2}}}\left[C_{1}\left[\ln \left(\frac{t+C_{2}+\sqrt{\left(t+C_{2}\right)^{2}+C_{1}^{2}}}{C_{1}}\right)\right]+C_{3}\right]-C_{1}
\end{aligned}
$$


Now we show the modulus of the control is 1 . For that we have,

$$
\begin{aligned}
& u_{1}^{2}+u_{2}^{2} \\
& =\left(t+C_{2}\right)^{2}+\frac{C_{1}^{2}}{\left(t+C_{2}\right)^{2}+C_{1}^{2}}\left[C_{1}\left[\ln \left(\frac{t+C_{2}+\sqrt{\left(t+C_{2}\right)^{2}+C_{1}^{2}}}{C_{1}}\right)\right]+C_{3}\right]^{2} \\
& +\frac{\left(t+C_{2}\right)^{2}}{\left(t+C_{2}\right)^{2}+C_{1}^{2}}\left[C_{1}\left[\ln \left(\frac{t+C_{2}+\sqrt{\left(t+C_{2}\right)^{2}+C_{1}^{2}}}{C_{1}}\right)\right]+C_{3}\right]^{2}+C_{1}^{2} \\
& =\left(t+C_{2}\right)^{2}+C_{1}^{2}+\left[C_{1}\left[\ln \left(\frac{t+C_{2}+\sqrt{\left(t+C_{2}\right)^{2}+C_{1}^{2}}}{C_{1}}\right)\right]+C_{3}\right]^{2} \\
& =q_{1}^{2}+q_{2}^{2} \\
& =1 \text { by equation 25. }
\end{aligned}
$$

This shows that the norm of the control is 1 which should always be the case when the normal extremals are arc length parametrized.

\section{Computation Of the Curvatures}

We know $u_{1}^{2}+u_{2}^{2}=1$. So we can define $u_{1}=\cos (\theta)$ and $u_{2}=\sin (\theta)$ for some $\theta$. Then considering a $(q, \theta)$ coordinate the following equations can be written down as shown in [5]

$$
\begin{aligned}
& \dot{\theta}=c_{1}(q) \cos (\theta)+c_{2}(q) \sin (\theta) \\
& \dot{q}=\cos (\theta) f_{1}(q)+\sin (\theta) f_{2}(q)
\end{aligned}
$$

where $c_{1}, c_{2} \in C^{\infty}(M)$ such that [5]

$$
\left[f_{1}, f_{2}\right]=c_{1} f_{1}+c_{2} f_{2}
$$


This suggests that an arc length parametrized normal extremal(that is which satifies the second equation of the set (56)) satisfies the first equation of the set (56). This suggests that the geodesic curvature of the trajectory on $M$ can be written as $[\underline{5}$

$$
\kappa_{g}=\dot{\theta}-c_{1}(q) \cos (\theta)-c_{2}(q) \sin (\theta)
$$

Now we need to compute the functions $c_{1}$ and $c_{2}$. For that we need to compute the Lie bracket $\left[f_{1}, f_{2}\right]$. If $X_{1}, X_{2}$ are the components of $f_{1}$ and $Y_{1}, Y_{2}$ are the components of $f_{2}$, denoting $\frac{\partial}{\partial q_{1}}$ by $\partial_{1}$ and $\frac{\partial}{\partial q_{2}}$ by $\partial_{2}$ first component of the vector $\left[f_{1}, f_{2}\right]$ is given by

$$
\begin{aligned}
& X^{1} \partial_{1} Y^{1}-Y^{1} \partial_{1} X^{1}+X^{2} \partial_{2} Y^{1}-Y^{2} \partial_{2} X^{1} \\
& =0-q_{2}+q_{2}-0 \\
& =0
\end{aligned}
$$

second component of the vector $\left[f_{1}, f_{2}\right]$ is given by

$$
\begin{aligned}
& X^{1} \partial_{1} Y^{2}-Y^{1} \partial_{1} X^{2}+X^{2} \partial_{2} Y^{2}-Y^{2} \partial_{2} X^{2} \\
& =-q_{1}-0+0+q_{1} \\
& =0
\end{aligned}
$$

Therefore from equation (57),

$$
\begin{gathered}
0=\left[f_{1}, f_{2}\right]=c_{1} f_{1}+c_{2} f_{2} \\
\Longrightarrow c_{1}=c_{2}=0
\end{gathered}
$$


Now we have considered $u_{1}=\cos (\theta)$. Therefore $\theta=\cos ^{-1}\left(u_{1}\right)$. Therefore,

$\theta=\cos ^{-1}\left[t+C_{2}+\frac{C_{1}}{\sqrt{\left(t+C_{2}\right)^{2}+C_{1}^{2}}}\left[C_{1}\left[\ln \left(\frac{t+C_{2}+\sqrt{\left(t+C_{2}\right)^{2}+C_{1}^{2}}}{C_{1}}\right)\right]+C_{3}\right]\right]$

Therefore,

$$
\dot{\theta}=-\frac{1-\frac{C_{1}\left(t+C_{2}\right)}{\left[\left(t+C_{2}\right)^{2}+C_{1}\right]^{\frac{3}{2}}}\left[C_{1}\left[\ln \left(\frac{t+C_{2}+\sqrt{\left(t+C_{2}\right)^{2}+C_{1}^{2}}}{C_{1}}\right)\right]+C_{3}\right]+\frac{C_{1}^{2}}{\left(t+C_{2}\right)^{2}+C_{1}^{2}}}{\sqrt{1-\left[t+C_{2}+\frac{C_{1}}{\sqrt{\left(t+C_{2}\right)^{2}+C_{1}^{2}}}\left[C_{1}\left[\ln \left(\frac{t+C_{2}+\sqrt{\left(t+C_{2}\right)^{2}+C_{1}^{2}}}{C_{1}}\right)\right]+C_{3}\right]\right]^{2}}}
$$

Also $c_{1}=c_{2}=0$.

Therefore by equation (58) the geodesic curvature,

$$
\kappa_{g}=-\frac{1-\frac{C_{1}\left(t+C_{2}\right)}{\left[\left(t+C_{2}\right)^{2}+C_{1}\right]^{\frac{3}{2}}}\left[C_{1}\left[\ln \left(\frac{t+C_{2}+\sqrt{\left(t+C_{2}\right)^{2}+C_{1}^{2}}}{C_{1}}\right)\right]+C_{3}\right]+\frac{C_{1}^{2}}{\left(t+C_{2}\right)^{2}+C_{1}^{2}}}{\sqrt{1-\left[t+C_{2}+\frac{C_{1}}{\sqrt{\left(t+C_{2}\right)^{2}+C_{1}^{2}}}\left[C_{1}\left[\ln \left(\frac{t+C_{2}+\sqrt{\left(t+C_{2}\right)^{2}+C_{1}^{2}}}{C_{1}}\right)\right]+C_{3}\right]\right]^{2}}}
$$

Note that the geodesic curvature depends on time $t$.

The Gaussian curvature is given by $[\underline{5}$,

$$
\kappa=f_{1}\left(c_{2}\right)-f_{2}\left(c_{1}\right)-c_{1}^{2}-c_{2}^{2}
$$

Now $c_{1}=c_{2}=0$. Therefore $f_{1}\left(c_{2}\right)=f_{2}\left(c_{1}\right)=0$ which implies the gaussian curvature,

$$
\kappa=0
$$

which should be case when $f_{1}, f_{2}$ are involutive that is their Lie bracket generates 0. 


\section{Conclusion}

We try to conclude the article with a modest conclusion. We want to raise the important points of our work. First thing we want to point out is about the nature of optimality that we have considered in our work. What we have done is actually spacial optimality and not time optimality as time is fixed between 0 and 1 . In this constraint we obtained the normal extremals exhibited by the trajectory using Pontryagin Minimization formalism. The normal extremals are supposed to be the geodesic on the manifold. The Hamilton's equations that came up in this regard generated nonlinear differential equations of time which are hard to solve. But using the fact that normal extremals are parametrized by arc length which in turn implies the velocity at each point of the trajectory is 1 , we were able to solve the equations successfully. The optimal controls were solved with respect to time $t$. We further showed that the modulus of the control is 1 which is always the case in case of arc length parametrized extremals. Further we computed the geodesic curvature and the Gaussian curvature of the Riemannian structure. The geodesic curvature is found to depend on time $t$ while the Gaussian curvature evaluates to 0 . We wish to comment about the result of Gaussian curvature that we found. The reason for that, the orthogonal vector fields which we have considered for our work are found to be involutive that is their Lie Bracket generates 0. The curvature is always 0 in the case when the family of vector fields is involutive.

\section{References}

[1] Héctor J Sussmann. New differential geometric methods in nonholonomic path finding. In Systems, models and feedback: theory and applications, pages 365-384. Springer, 1992. 
[2] Yacine Chitour. A continuation method for motion-planning problems. ESAIM: Control, Optimisation and Calculus of Variations, 12(1):139-168, 2006.

[3] Yacine Chitour and Héctor J Sussmann. Line-integral estimates and motion planning using the continuation method. In Essays on Mathematical Robotics, pages 91-125. Springer, 1998.

[4] Hector Sussmann. A continuation method for nonholonomic path-finding problems. In Proceedings of 32nd IEEE Conference on Decision and Control, pages 2718-2723. IEEE, 1993.

[5] Andrei Agrachev, Davide Barilari, and Ugo Boscain. A comprehensive introduction to sub-Riemannian geometry, volume 181. Cambridge University Press, 2019. 